\title{
A Case of Normocomplementemic Urticarial Vasculitis Triggered by Urinary Tract Infection
}

\author{
Esra DEMİR, Özgül Mustu KORYÜREK², Onur TANRIKULU ${ }^{1}$ \\ ${ }^{1}$ Istanbul Medipol University Mega Hospital, Department of Internal Medicine, Istanbul, Turkey \\ ${ }^{2}$ Turkish Hospital, Dermatology Clinic, Doha, Qatar
}

\begin{abstract}
Urticarial vasculitis is a variant of cutaneous small-vessel vasculitis. Urticarial vasculitis is generally classifiedintotwotypes: normocomplemanaemicurticarial vasculitis and hypocomplementemiaurticarial vasculitis. Here, we presented a case with a diagnosis of normocomplementemic urticarial vasculitis.
\end{abstract}

Turk J Int Med 2021;3(Supplement 1):S57-S59 DOI: $10.46310 /$ tjim. 875858

Keywords: Urticaria, Vasculitis, Normocomplementemia

\section{Introduction}

Urticarial vasculitis (UV) can be both classified under the heading of urticaria and considered a subtype of leukoclastic vasculitis, characterized by urticarial lesions. Histopathologically, it is distinguished from simple urticaria by the presence of vascular injury.1 Its typical findings include lesions lasting for more than 24 hours that do not blanch upon pressure; burning and pain being the predominant symptoms rather than itching; lesions that leave pigmentation when fading; a skin biopsy showing endothelial swelling and injury usually involving postcapillary venules, secondary red blood cell extravasation, fragmentation of leukocytes (leukocytoclasis), fibrin deposition in the vessel wall, and perivascular infiltration with a neutrophil predominance. ${ }^{1}$
In addition, it is possible to show immunoglobulin, complement, or fibrin deposits in the perivascular area and/or dermo-epidermal junction by direct immunofluorescent (DIF) examination. ${ }^{2}$ Although these findings are not typical of UV, their absence does not exclude the condition, either. The underlying cause is sometimes drugs, stress, malignancies, rheumatological disorders, autoimmune disorders; occasionally, an infection is the culprit trigger, as is the case in our patient. ${ }^{3}$

\section{Case Report}

A 39-year-old woman, who had no history of any disease other than urticaria and who had been occasionally using antihistaminic medications for

Received:February 07,2021;Accepted:March 3,2021; Published Online:March 6, 2021

Istanbul Medipol University Mega Hospital, Department of Internal Medicine, Istanbul, Turkey 

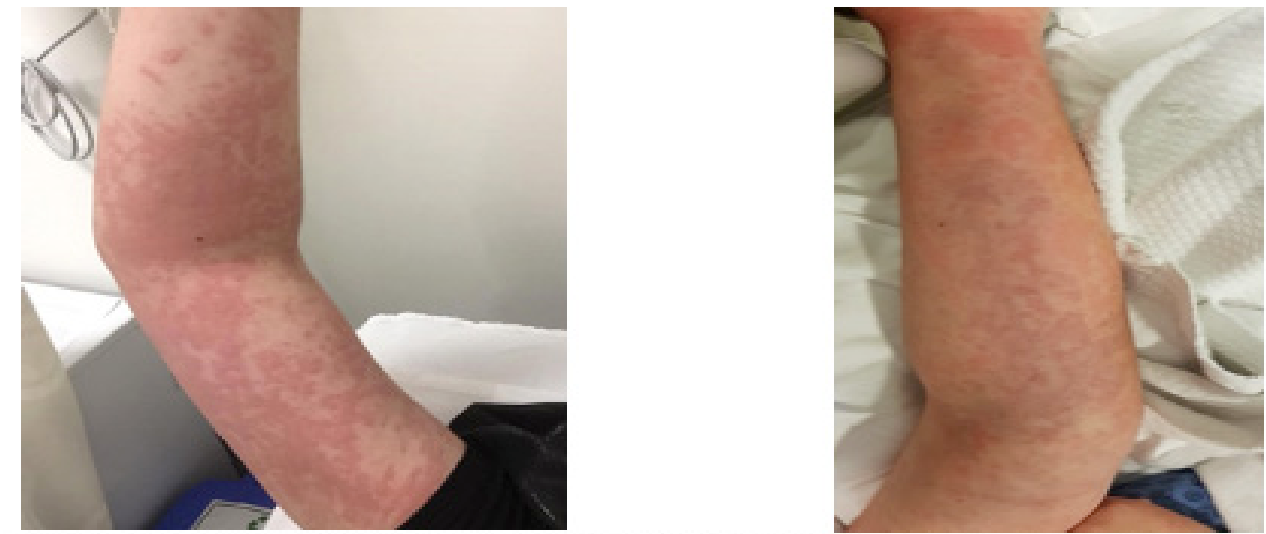

Figure 1 and 2. Widespread urticarial plaques and purple lesions that faded in patches all over the body

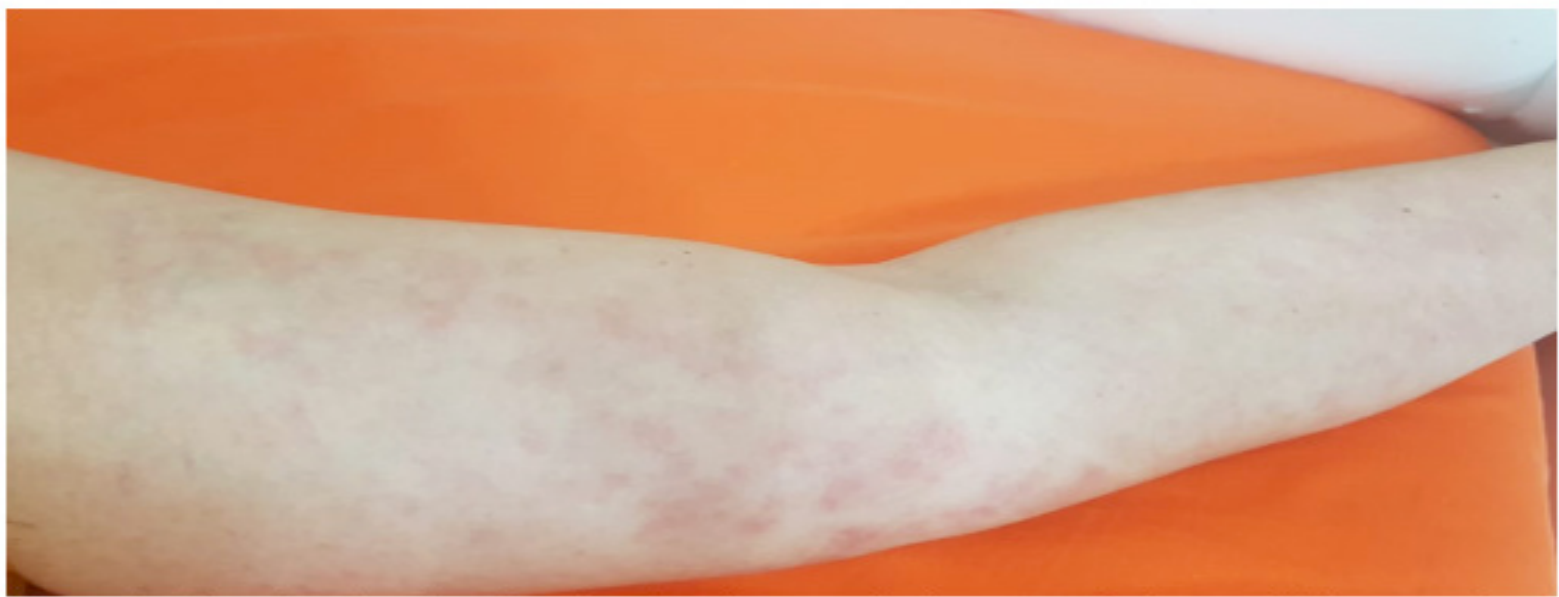

Figure 3. The patient's rash and angioedema have gradually regressed, the steroid dose was down titrated

itching, presented to our outpatient clinic with a diffuse rash all over the body, itching, and difficulty breathing. On physical examination, she had edema and redness of the entire face, which were more prominent on the eyelids and lips, as well as widespread urticarial plaques and purple lesions that faded in patches all over the body (Figure 1 and 2). She had no uvula edema or abnormal breath sounds. Her history was not notable for any medication except for an antihistaminic medication that she had taken in the last month; her complaints had started 5 days ago and not responded to the antihistaminic medications (rupatadine fumarate) that she had used. It was also learned that she had been admitted to the emergency service of an outside medical facility, where she had been treated with intravenous pheniramine, methylprednisolone, and dexamethasone; her complaints had regressed following the first three days of intravenous treatment but resumed thereafter. As she began to have a skin rash, itching, burning, and stinging sensation again on the fourth day, and as she remained unresponsive to intravenous treat- ment administered in the emergency service, she presented to our facility with resistant urticaria. Her laboratory tests revealed the following: urea $39 \mathrm{mg} / \mathrm{dL}$, creatinine $0.81 \mathrm{mg} / \mathrm{dL}$, total protein 76 $\mathrm{g} / \mathrm{L}$, albumin $45.5 \mathrm{~g} / \mathrm{L}$, uric acid $2.7 \mathrm{mg} / \mathrm{dL}, \mathrm{AST}$ 13 IU/L, ALT 15 IU/L, LDH 258I U/L (N: 135214), ALP 52I U/L, GGT 10 IU/L, total bilirubin $0.47 \mathrm{mg} / \mathrm{dL}$, direct bilirubin $0.15 \mathrm{mg} / \mathrm{dL}$, calcium $9.5 \mathrm{mg} / \mathrm{dL}$, sodium $140 \mathrm{mmol} / \mathrm{L}$, potassium $4 \mathrm{mmol} / \mathrm{L}$, lekocyte $22.2 \times 103 \mathrm{mcg} / \mathrm{L}$, neutrophil $20.02 \times 103 \mathrm{mcg} / \mathrm{L}$, lymphocyte $0.95 \times 103 \mathrm{mcg} / \mathrm{L}$, monocyte $1.21 \times 103 \mathrm{mcg} / \mathrm{L}$, TSH $1.38 \mathrm{mlU} / \mathrm{L}$, HBsAg, anti-HCV and anti-HIV were negative. Anti TPO and anti-thyroglobulin were negative. Sedimentation rate $10 \mathrm{~mm} / \mathrm{h}$, C3 $1.15 \mathrm{~g} / \mathrm{L}(\mathrm{N})$, C4 $0.14 \mathrm{~g} / \mathrm{L}(\mathrm{N})$, total IgE $296 \mathrm{mcg} / \mathrm{L}$ (N: 0-240), CRP $15 \mathrm{mg} / \mathrm{L}$. In urinalysis, there were 7 erythrocytes and 7 leucocytes.

The patient was admitted to a regular ward with the diagnoses of resistant urticaria and urinary tract infection. Urine culture, PA chest X-Ray, ANA, and Anti-ds DNA were ordered. ANA and Anti-ds DNA tests returned negative. 
There were no pulmonary infiltrates on PA chest X-Ray. The patient was begun on intravenous methylprednisolone at a dose of $1 \mathrm{mg} / \mathrm{kg}$, pheniramine, and pantoprazole. Empirical intravenous ceftriaxone was also added for a possible urinary tract infection. As urticarial lesions persisted for more than 24 hours and they left pigmentation after fading and caused pain and tingling sensation in addition to itching, UV was primarily considered, which prompted us to take a punch biopsy of $4 \mathrm{~mm}$ thick from the lesions. As the patient's rash and angioedema have gradually regressed, the steroid dose was down titrated (Figure 3). Urine culture produced no bacterial proliferation. The pathology report of the biopsied lesions returned as the following: mild orthokeratosis in the epidermis; edema and vascular proliferation in the upper dermis; swollen endothelium; perivascular infiltration by lymphocytes, histiocytes, neutrophils, and eosinophils; and the presence of neutrophils and interstitial eosinophils on some vascular walls. Diagnosed with "Normocomplementemic UV" that was triggered by a urinary infection, the patient was discharged to receive an oral cephalosporin, an antihistaminic, prednisolone, and urticarial diet.

\section{Discussion}

Our patient was a 39-year-old female with chronic urticaria, who had been having occasional urticaria attacks and antihistaminic use, but no chronic medication uses between the attacks. After having dysuria not mentioned as a complaint as well as starting to have a rash and itchy lesion that had been considered as her previous attacks but remained unresponsive to treatment first with oral antihistaminic medications and then daily intravenous steroids and antihistaminic medications, the patient presented to our medical institution for further investigation and treatment. As the patient had lesions that had persisted longer than those of simple urticaria, that had a color close to violet, and that left noticeable brown pigmentation when they faded; and additionally, as she had urinary tract infection and a high
CRP level, she was considered to have resistant urticaria caused by a urinary tract infection and $\mathrm{UV}$ as the preliminary diagnoses; therefore, C3 and $\mathrm{C} 4$ levels were studied and a skin biopsy was taken. C3 and C4 levels returned normal, and the skin biopsy was reported to be consistent with urticarial vasculitis. The patients were put on intravenous empirical antibiotic treatment after urine culture was taken; following the second antibiotic dose, the itching, burning, and stinging sensations began to subside, and the lesions began to fade. On the third day of treatment, the patient's lesions faded with mild residual pigmentation; itching and burning sensations completely disappeared; the CRP level returned to normal, and the urine culture revealed no bacterial proliferation. The patient was diagnosed with normocomplementemic urticarial vasculitis; subsequently, her treatment was arranged, and she was discharged with recommendations.

In patients with resistant urticarial symptoms who are unresponsive to classical urticaria treatment, underlying causes should be sought, and UV should be excluded, as was the case with our patient.

\section{Conflict of Interests}

Authors declare that there are none.

\section{Acknowledgment}

This study has been presented in $17^{\text {th }}$ Uludag Internal Medicine National Winter Congress, $6^{\text {th }}$ Bursa Family Medicine Association National Congress, $11^{\text {th }}$ Uludag Internal Medicine Nursing Congress, 5-7 March 2021, Bursa, Turkey.

\section{References}

1. Davis MD, Daoud MS, Kirby B, Gibson LE, Rogers RS 3rd. Clinicopathologic correlation of hypocomplementemic and normocomplementemic urticarial vasculitis. J Am Acad Dermatol. 1998 Jun;38(6 Pt 1):899-905.

2. Manappallil RG, Pallivalappil B, Martin AM, Mampilly N, Rao A. Normocomplementemic urticarial vasculitis: an unusual presentation. Indian J Dermatol. 2020 MayJun;65(3):208-10. doi: 10.4103/ijd.IJD_227_18.

3. Baigrie D, Bansal P, Goyal A, Crane JS. Leukocytoclastic Vasculitis [Updated 2020 Aug 11]. In: StatPearls [Internet]. Treasure Island (FL): StatPearls Publishing; 2021 Jan-. Available at: https://www.ncbi.nlm.nih. gov/books/NBK482159/. Accessed January 01, 2021. 